\title{
Germanica
}

\section{L'Allemagne dans la spirale du temps}

PolyPlay, roman policier uchronique de Marcus Hammerschmitt

Deutschland in der Spirale der Zeit. PolyPlay, ein Krimi über alternative

Geschichtserzählung von Marcus Hammerschmitt

Germany in a time vortex. PolyPlay, a cyber-thriller by Marcus Hammerschmitt

Hélène Yèche

\section{(2) OpenEdition}

Journals

Édition électronique

URL : http://journals.openedition.org/germanica/3195

DOI : $10.4000 /$ germanica.3195

ISSN : 2107-0784

Éditeur

Université de Lille

\section{Édition imprimée}

Date de publication : 30 septembre 2016

Pagination : 121-131

ISBN : 9782913857377

ISSN : 0984-2632

Référence électronique

Hélène Yèche, "L'Allemagne dans la spirale du temps », Germanica [En ligne], 58 | 2016, mis en ligne le 30 septembre 2018, consulté le 06 octobre 2020. URL : http://journals.openedition.org/germanica/ 3195 ; DOI : https://doi.org/10.4000/germanica.3195 


\title{
L'Allemagne dans la spirale du temps.
}

\author{
PolyPlay, roman policier uchronique \\ de Marcus Hammerschmitt
}

\author{
Hélène YÈCHE \\ Université de Poitiers
}

La césure provoquée par la dynamique unificatrice de l'automne 1989 dans l'histoire de l'Allemagne contemporaine représente une rupture paradoxale puisqu'elle conduisait dans le même temps à l'unité de la nation. Il s'agit néanmoins d'une rupture, comme en témoigna dès le début des années 2000 l'émergence du courant " ostalgique » (nostalgie du passé est-allemand ${ }^{1}$ ) - rupture suffisamment importante pour être le point de départ d'un polar uchronique intitulé PolyPlay, publié en 2002 par un jeune auteur allemand de science-fiction, Marcus Hammerschmitt, né en 1967 à Tübingen (RFA) ${ }^{2}$.

Situé au mois d'avril de l'an 2000, PolyPlay s'inscrit d'emblée dans l'histoire et la gestion du passé récent de la réunification de l'Allemagne : dix ans après la chute du mur de Berlin, la RDA a absorbé l'ancienne

1. - Thomas Ahbe, «Insofern ist Ostalgie nicht nur Nostalgie. Sie ist eine Art Selbsttherapie nach dem Umbruchs-Schock und ein laienhafter Versuch, sowohl die Spannung zwischen Vergangenheit und Gegenwart wie auch die zwischen der Selbstdeutung und der dominierenden Fremddeutung zu verhandeln », in : « Arbeit am kollektiven Gedächtnis. Die Fernseh-Shows zur DDR », Deutschland Archiv, November 2003, p. 917-924.

2. - Marcus Hammerschmitt, PolyPlay, Hamburg, Argument Verlag, 2002. Voir aussi http://www.marcus-hammerschmitt.de/ (page consultée le 15 octobre 2015). 
République fédérale ; contre toute attente, le modèle socialiste semble avoir fait la preuve de sa performance, tout au moins au sein de cet univers d'anticipation qui imagine de manière saisissante les possibles bouleversements consécutifs à la fin de la guerre froide.

Que penser de ce cyberthriller dont l'enquête plonge le lecteur dans une altération radicale de l'histoire récente ? S'inscrivant dans la lignée du genre cyberpunk, mouvement littéraire autour d'un monde futuriste ultra-informatisé, la virtualité informatique occupe une place majeure dans le roman de Hammerschmitt, non seulement en raison de l'enquête menée par la police est-allemande sur un jeu vidéo, mais également parce que l'intrigue se joue à différents niveaux de réalité, dont le premier est une plate-forme de serveurs informatiques en mer du Nord. Fondé sur un récit de mondes parallèles, puisque l'univers présenté comme référent au départ s'avère au final être celui d'un jeu vidéo ${ }^{3}$, PolyPlay semble parfaitement répondre à la définition de l'uchronie, genre peu prisé en littérature sauf par la science-fiction. Terme rare, forgé en 1867 par le philosophe français Charles Rénouvier sur le modèle de l'utopie, l'uchronie désigne ce qui n'existe en aucun temps. L'uchronie est ce pays imaginaire qui parle de ce qui n'a pas été tel qu'il aurait pu être. Comme le rappelle E. Carrère dans un essai consacré à ce genre méconnu, on entre en Uchronie par un scandale 4 . De ce point de vue, le roman policier de $\mathrm{M}$. Hammerschmitt semble bien répondre au principe premier du récit uchronique puisqu'il adopte pour prémisses une distorsion temporelle sans précédent et, de ce fait, bouleverse considérablement notre perception de l'histoire récente : l'argument de départ, la victoire historique, politique et économique de la RDA sur la RFA, pouvant sans conteste être assimilé à une relecture scandaleuse du passé de l'Allemagne.

\section{1- Lecture historique}

Pourtant, ce scandale n'est pas déclaré d'emblée comme tel. Il n'y a pas d'avant-propos à la fois tonitruant et didactique, soucieux d'éclairer le lecteur sur les intentions uchroniques et, partant, révolutionnaires

3. - Le jeu vidéo « Polyplay » a bel et bien existé : il s'agit de l'unique jeu d'arcade du marché est-allemand, produit à 1000 exemplaires environ, commercialisé dès 1986 en RDA et présent dans de nombreux centres pour la jeunesse. Cette machine, qui pouvait rapporter jusqu'à 5000 marks par jour, devait son nom autant à son créateur, le consortium VEB Polytechnik de Karl Marx Stadt, qu'au fait qu'elle permettait de jouer à 8 jeux différents comme l'explique Andreas Lange, directeur du Musée des jeux informatiques et vidéo à Berlin, « Computer- und Videospiele in der DDR », 16.06.1998, http://www.heise.de/tp/artikel/3/3249/1.html (page consultée le 15 octobre 2015). Marcus Hammerschmitt s'est inspiré de cet article pour écrire son roman PolyPlay.

4. - Emmanuel Carrère, Le Détroit de Behring, Paris, P.O.L., 1986, p. 9. 
de l'auteur. Le roman débute comme un polar au scénario classique : il s'ouvre sur le quotidien morose et glacé d'un policier enquêtant sur la mort violente d'un jeune garçon. Mais dès ce premier chapitre, au détour d'une phrase, l'attention du lecteur est captée par l'utilisation d'un vocabulaire spécifique au passé est-allemand : à l'occasion de l'entrée en scène d'un policier, l'emploi de l'acronyme « Vopo », dérivé du terme allemand Volkspolizist, et qui ne saurait donc renvoyer qu'à la police nationale de l'ancienne RDA, suscite la curiosité. Le ton est donné grâce à l'utilisation d'un langage théoriquement disparu, signe du décalage temporel sur lequel est fondé le récit. La mention, quelques lignes plus loin, de la FDJ, la Jeunesse allemande libre, organisation de masse destinée à encadrer les plus jeunes d'un point de vue idéologique dans le cadre de l'ancienne RDA, vient confirmer le malaise du lecteur. Le titre de ce premier chapitre, ironiquement intitulé «Kopfweh» (Mal de crâne), instaure un climat de complicité entre lecteur et enquêteur qui renforce d'emblée l'empathie du premier.

La présence de ces sigles typiquement est-allemands, appartenant en théorie à un passé révolu, résonne comme le véritable signal de l'entrée en Uchronie. Quelques pages encore et l'évidence s'impose : nous sommes en RDA, au matin du 3 avril 2000 (PolyPlay, p. 12). L'enquête policière se poursuivant, le lecteur n'a d'autre choix que de suivre le lieutenant Kramer, personnage principal de l'enquête, espérant en apprendre un peu plus lui aussi sur ce dérèglement du temps, si bien qu'une double intrigue se noue dans ce polar uchronique : à l'enquête administrative du policier se superpose celle, historique, du lecteur dont les repères chronologiques sont bouleversés. Enfin, au bout d'une dizaine de pages, après plusieurs mentions anodines du contexte de la «nouvelle RDA », l'adjectif «nouveau » insistant bien sur l'accomplissement d'un changement à l'échelle du temps historique, l'incroyable s'impose au lecteur : le tournant, la Wende a bien eu lieu comme l'indique clairement le titre du deuxième chapitre «Wieder vereint », Réunifié.

Ce premier chapitre, capital pour la mise en situation de l'uchronie, vise à replacer l'action dans un cadre historique connu: celui de la fin des années 1980 et du mécontentement grandissant au sein du bloc de l'Est. À la politique libérale de Gorbatchev, la RDA répond, comme dans la réalité historique, par une ligne encore plus stricte ; le mécontentement de la population est-allemande, sensible aux sirènes du capitalisme, prépare le terrain au changement. Mais le constat laconique formulé à la fin du paragraphe historique : « et alors eut lieu la Wende » (PolyPlay, p. 20), laisse encore une fois le lecteur sur sa faim. L'enquête reprend le dessus tandis que l'on découvre au fil du texte les détails de ce nouvel environnement socialiste dans lequel la Stasi occupe toujours les immeubles de brique rouge de la Ruschestraße, mais s'est modernisée, s'emparant de l'Internet pour en faire un Internetz sur le modèle 
des Intershop (les magasins réservés aux cadres du parti du temps de la RDA historique) ; cette nouvelle RDA tolère même, comble du renversement historique pour le lecteur, une certaine nostalgie de la RFA de la part des Wessis. Au chapitre 4 intitulé «Identifikation » (Identification), on apprend de la bouche de Doernberger, un ancien résistant, communiste de la première heure, ce qui s'est réellement passé : c'est l'Ouest qui a réclamé son rattachement au système communiste après l'effondrement du capitalisme, et le gouvernement de Hans Modrow, moins ferme que celui d'Honecker en son temps, a eu la faiblesse d'ouvrir le Mur pour permettre aux Wessis d'envahir l'Allemagne de l'Est. À la suite de ce récit, qui pointe sans ménagement les défaillances et les contradictions du nouveau «pluralisme socialiste », le lecteur est aussi abasourdi que Kramer, à qui cette rencontre semble avoir révélé la vraie nature de la réunification allemande. Il faut ensuite attendre le chapitre 6 pour en apprendre un peu plus sur le déroulement de cette unification à l'envers, qui fut l'occasion pour la RDA d'une modernisation et d'un renouveau sans précédent. Le reste du temps, l'enquête policière progresse lentement, émaillée de sigles jargonneux plus vrais que nature ${ }^{5}$. Ainsi l'uchronie s'impose-t-elle progressivement au lecteur comme une évidence tant les détails sont nombreux et soignés, et surtout de l'ordre du vraisemblable. Passé le choc initial de l'altération, une fois surmontée l'idée de l'impensable, on s'interroge logiquement sur le pourquoi d'un tel choix narratif.

\section{2- Lecture politique}

Certes l'uchronie permet d'interroger la façon dont nous percevons le passé, elle permet de reconsidérer l'importance d'événements achevés en les présentant sous un jour radicalement nouveau. Mais elle demeure une imposture dans l'Histoire, une falsification scandaleuse puisqu'on ne révoque pas l'irrévocable, même par le pouvoir des mots. Pourtant l'uchroniste travaille rarement sans arrière-pensée : quel objectif sert donc ici l'uchronie ?

En dépit de prémisses incroyables, l'univers de la fiction ne se révèle tout d'abord ni plus cauchemardesque, ni plus idyllique que la réalité historique. Il n'y a ni message politique, ni dénonciation de la dictature socialiste - même si PolyPlay est bien une critique de la RDA décrite par l'auteur comme « une entreprise très étrange, un socialisme organisé, non révolutionnaire dans une société post-fasciste ${ }^{6}$.

5. - M. Hammerschmitt, qui s'est longuement documenté sur le système socialiste, affirme que la plupart des sigles employés sont authentiques, à l'exception d'une ou deux inventions personnelles comme « NATA » par exemple (Entretien avec H. Yèche, mars 2007 - non publié).

6. - «Zweitens kritisiert Polyplay die DDR. Eigentlich war sie ja ein sehr 
Pas de dyschronie donc, ni d'apologie d'un système qui semble avoir évolué, mais dont les déficits demeurent épinglés, comme en témoigne la scène implacable dans un établissement d'enseignement où Kramer note qu'on débite toujours les mêmes salades (PolyPlay, p. 71). PolyPlay n'est donc pas une utopie marxiste, ce n'est pas un récit de politique fiction. Ce qui a motivé l'écriture de ce polar, c'est à la fois « l'arrogance des vainqueurs et l'aveuglement des nostalgiques de la RDA qui croient encore aujourd'hui que tout était merveilleux en RDA. » L'auteur fait le constat en 2000 «que la RDA est d'une part muséalisée, idéalisée, et d'autre part diabolisée, et qu'elle disparaît dans ce curieux processus de conservation pour gagner un monde fantastique dont on ne peut dire s'il a existé ». Ainsi l'auteur a-t-il cherché « à recréer la fiction d'une RDA qui n'a jamais existé » tout en déclarant : «J'estime être un auteur politique, mais je ne m'installe pas à ma table de travail avec des objectifs politiques précis ${ }^{7}$. Si M. Hammerschmitt revendique une écriture politique, l'auteur ne semble pas fonder son uchronie sur un désir compensatoire et, en ce sens, on perçoit déjà la distance qui s'instaure petit à petit avec le modèle littéraire.

\section{3- Lecture parodique}

Car l'écriture dans PolyPlay est bien trop ironique et parodique pour être militante. Mais c'est précisément en cela que réside sa force comme l'explique l'auteur, à condition toutefois d'éviter le prosélytisme :

La SF a le pouvoir «d'aller là où aucun autre genre ne va », et elle peut indiquer des clairières dans la forêt que les arbres ont fini par cacher. Le grand art consiste alors à éviter l'agitprop pour obéir à l'éthique fondamentale du narrateur : raconter une histoire intéressante aux lecteurs. La propagande est capable de forger des slogans au quotidien, mais dans un roman, elle ne provoque que l'ennui. ${ }^{8}$

seltsames Unterfangen, ein veranstalteter, nicht-revolutionärer Sozialismus in einer postfaschistischen Gesellschaft ». Jakob Schmidt, « Eine Säure, die den ganzen Müll wegätzt », in : Alien Contact. Nr. 51, 19. Februar 2003 (Interview mit Marcus Hammerschmitt).

7. - « Motiviert haben mich verschiedene Dinge. Die Arroganz der Sieger und die Realitätsfremdheit der DDR-Nostalgiker, die ja heute noch glauben, dass in der DDR alles wunderbar war. [...] Und nun stellte ich im Jahr 2000 fest, dass sie einerseits musealisiert und idealisiert, andererseits immer noch dämonisiert wurde, und dass sie in diesem seltsamen Prozess der Aufbewahrung gleichzeitig verschwand, in eine Phantasiewelt verwandelt wurde, von der man nicht sagen konnte, inwiefern ihr je Realität zugekommen war. Dem wollte ich beikommen mit der Phantasie von einer DDR, wie sie nie gewesen ist. [...] Ich verstehe mich als politischen Autor, aber ich setze mich nicht mit bestimmten politischen Zielen an den Schreibtisch » (Entretien avec H. Yèche, op. cit.).

8. - «Die SF kann 'gehen, wohin kein anderes Genre geht', und sie kann 
Pour M. Hammerschmitt, la science-fiction se rapproche de la satire, mais une satire sans conciliation finale :

La science-fiction [...] n'est pas une forme traditionnelle de satire qui fonctionnerait comme instance morale sous un habillage humoristique, mais elle déforme son objet en grossissant le trait jusqu'à ce qu'on puisse le reconnaître, sans tabler sur l'effet de délivrance et de réconciliation que provoque le rire 9 .

L'auteur cherche également à ébranler le mode de réception classique du roman qui repose sur l'identification avec le héros. Flanqué de son acolyte Pasulke, le personnage de Kramer, solitaire au cœur pur, incarne une figure pathétique inspirée des séries B, qui doit forcer l'identification pour mieux provoquer chez le lecteur le choc final que l'auteur lui réserve au moment où il découvre la conversion radicale du héros supposé10.

La plupart des chapitres du roman portent d'ailleurs un titre dont la portée dépasse le cadre strict de la fiction : l'intertextualité qui se dégage de ces titres participe du caractère éminemment ludique de l'écriture de M. Hammerschmitt. Wieder vereint, Identifikation sont des titres à double sens, mais à signification interne. L'intertextualité est parfois extra-diégétique comme dans le cas suivant : Der Akkermann und der Tod, littéralement : Le Laboureur et la mort. Ce titre fonctionne comme citation parodique, en l'occurrence il s'agit d'une allusion à l'œuvre de Johannes Tepl, un des premiers témoignages de l'humanisme allemand composé vers 1400 qui raconte le désespoir d'un laboureur dont l'épouse vient de mourir et qui s'adresse à la mort dans un dialogue remarquable de revendications humanistes à une époque de toute-puissance de la religion. Mais ici la citation littéraire est sciemment détournée puisque la disparition de l'épouse est le fait d'un dénommé Akkermann (et non Ackermann comme dans l'œuvre originale) avec qui la femme de Kramer est partie...

Lichtungen in einem Wald aufzeigen, den viele Leute vor lauter Bäumen gar nicht mehr sehen. Die große Kunst dabei bleibt, Agitprop zu vermeiden und der grundsätzlichen Ethik des Erzählers zu gehorchen, nämlich den Lesern eine interessante Geschichte zu erzählen. Propaganda kann Tageslosungen bündeln, ödet aber als Roman nur an. » Interview de Marcus Hammerschmitt avec Jakob Schmidt, op. cit., p. 2.

9. - « Science Fiction [...] ist keine herkömmliche Form der Satire, die als moralische Anstalt mit humoristischer Garnitur funktioniert, sondern eine, die ihr Thema durch überzeichnende Verzerrung zur Kenntlichkeit entstellt, ohne auf den erlösend-versöhnenden Effekt des Gelächters zu spekulieren ». Marcus Hammerschmitt : «Zur Kenntlichkeit entstellt »- Interview über Science Fiction als unversöhnliche Satire, Phase 2, 06/2003, p. 62-63.

10. - Interview de Marcus Hammerschmitt avec Jakob Schmidt, op. cit., p. 2. 
Du point de vue de la composition du roman, le titre PolyPlay annonce d'emblée la mise en abyme fondamentale qui instaure le principe du jeu comme élément moteur de l'écriture. L'avant-dernier chapitre s'intitule d'ailleurs « Finale », ultime révélation du caractère ludique de l'intrigue et surtout de la partie qui est en train de se jouer à l'insu de tous, enquêteurs, lecteurs et personnages.

À l'affirmation initiale de l'impensable victoire du socialisme fait effectivement écho la démystification finale : tout était faux, apprendon en même temps que le protagoniste, « il n'y a plus de Chambre du peuple, plus de police nationale, plus de socialisme ${ }^{11}$. Pourtant, au lieu de déconstruire le schéma uchronique, cette révélation ne fait que le relancer. Loin de ramener personnages et lecteurs à un niveau de réalité rassurant - comme on pourrait le croire (ou le souhaiter) dans un premier temps -, l'auteur nous entraîne dans la spirale vertigineuse d'un temps dont la réalité se dérobe à l'infini. Dans ce final époustouflant, Kramer a beau s'exclamer « Mais que signifie tout cela ! / Was soll das ! », on lui réplique que cette « vie dans une RDA qui n'existe plus vraiment, qui n'a jamais existé telle quelle » ${ }^{12}$, n'est autre que la réalité, c'est-à-dire le niveau zéro du jeu.

Ainsi PolyPlay se révèle être bien plus qu'un simple jeu vidéo auquel s'adonnait l'adolescent dont Kramer doit élucider la mort... PolyPlay se découvre magistralement à la hauteur de la sémantique plurielle contenue dans le titre, puisque ce jeu vidéo fait partie d'un projet de recherche d'initiative privée, un jeu qui met en scène l'annexion de la RFA par la RDA et non l'inverse, et dans le cadre duquel le décor de l'ancienne RDA a été recréé électroniquement. PolyPlay, comme l'explique à la fin du roman l'un de ses concepteurs à Kramer, est un logiciel, expérimental et uchronique par excellence, qui sert à explorer les multiples scénarii de l'histoire selon le principe du « Et si / Was wäre, wenn » (PolyPlay, p. 174).

\section{4- Lecture ludique}

L'avant-dernier chapitre intitulé « Endspiel », Finale, n'est pas encore l'épilogue. Il s'ouvre cependant sur une déclaration inhabituelle en mode uchronique, censée signifier au lecteur le renversement radical de perspective : «Le monde marche sur la tête » ${ }^{13}$. Ce constat définitif auquel ne conduit pas habituellement la structure uchronique révèle brusquement au lecteur le processus de distanciation soigneusement élaboré par

11. - «Es gibt keine Volkskammer mehr, keine deutsche Volkspolizei und keinen Sozialismus », PolyPlay, op. cit., p. 173.

12. - «Das Leben in einer DDR, die es in Wirklichkeit nicht mehr gab. Die es so nie gegeben hatte », PolyPlay, ibid., p. 186.

13. - « Die Welt wurde umgestülpt», PolyPlay, ibid., p. 162. 
l'auteur. En réalité, nombre de récits uchroniques contiennent en avantpropos la trace du principe de réflexivité de l'écriture qui se présente comme consciente de la mise en abyme opérée. Le modèle littéraire s'émancipe ici des conventions pour garder intact le plaisir de la créativité narrative. L'auteur insiste d'ailleurs sur l'importance du récit qui prime à ses yeux sur la critique socio-politique :

D'ailleurs, et je ne saurais trop insister sur ce point, l'objectif premier de PolyPlay comme celui de tous mes textes narratifs n'était pas de critiquer (je réserve cela à mes essais), mais de raconter une histoire qui s'est imposée à moi. ${ }^{14}$

On trouve une allusion rapide à l'architecture enchâssée du roman dans le récit cadre constitué de sept chapitres imprimés en italiques et disséminés entre les chapitres du récit second. Ce récit premier situe l'action sur une plate-forme de la mer du Nord ${ }^{15}$ et présente la figure d'un jeune informaticien américain nommé Wes, responsable de la surveillance d'un parc d'ordinateurs puissants au sein duquel il découvre un dysfonctionnement qui introduit indirectement la structure narrative du roman par la mention du mot « histoires » : «Wes voulait découvrir de quel genre d'histoires il pouvait bien retourner $»^{16}$. Ce dysfonctionnement constitue la matière première du récit second, au sein duquel Kramer fait lui-même une allusion au principe narratif de l'uchronie en expliquant, dès les premières pages, qu'il a l'impression de se trouver dans une "machine à remonter le temps mal programmée », dans un « univers parallèle où il est contraint de ressasser les discours et toute la phraséologie qu'il avait dû ingurgiter jusqu'à l'écœurement durant ses jeunes années $\gg^{17}$. Mais en dehors de ce bref éclair de lucidité, le personnage ne soupçonne rien du jeu dont il est l'objet, jusqu'à la révélation finale. Kramer prend alors subitement conscience, en même temps que le lecteur, du caractère doublement artificiel de son existence puisqu'il n'est au fond qu'un pion au sein d'un jeu vidéo :

14. - « Ansonsten, und das kann ich nicht oft genug betonen, wollte ich mit PolyPlay wie mit all meinen erzählenden Texten nicht primär etwas kritisieren (das behalte ich meinen Essays vor), sondern eine Geschichte erzählen, die sich mir aufdrängte. » Interview de Marcus Hammerschmitt avec Jakob Schmidt, op. cit., p. 2.

15. - L'auteur s'est d'ailleurs inspiré ici d'un fait réel. Cf. Florian Rötzer, « Die künstliche Insel der freien Daten », Telepolis, 23.06.2002.

16. - « Wes wollte herausfinden, was das für Geschichten waren », PolyPlay, op. cit., p. 146.

17. - « Kramer kam sich vor wie in einer Zeitmaschine, die auf das falsche Datum eingestellt war, wie in einer Parallelwelt, in der er gezwungen wurde, die in seinen frühen Jahren bis zum Erbrechen mitangehörten Diskussionen und Phrasen noch einmal durchzukauen », PolyPlay, ibid., p. 47. 
Tu es un logiciel. Tu es un programme, Rüdiger, élaboré pour jouer à un jeu avec nous. Le jeu se nomme PolyPlay. Ce n'est pas le petit jeu stupide que tu as connu jusque là. [...] Il s'agit d'un jeu beaucoup plus important, beaucoup plus complexe, bien plus remarquable que tout ce que tu peux imaginer. C'est un univers. Plusieurs mondes. Nous sommes des joueurs. Nous sommes des dieux. Et tu fais partie du jeu. Tu es une figure intelligente et sensible du jeu. Une pièce du jeu d'échec dotée d'un cerveau ${ }^{18}$.

Le vertige qui saisit alors lecteur et personnage n'est que le prélude au feu d'artifice qui fait basculer l'intrigue policière et le roman d'anticipation vers la réflexion métaphysique.

La pirouette finale qui introduit le pseudo-retour au réel n'est qu'une ultime mise en abyme de la condition humaine. La révélation inattendue du statut de «software » du personnage principal joue encore sur le registre de la parodie, puisque les principaux interlocuteurs de Kramer ne sont autres que Karl Marx et Zeus. M. Hammerschmitt semble se complaire dans l'anachronisme « uchronique » qui permet de dénoncer l'hétéronomie du monde dans lequel nous vivons en critiquant les « mythes, l'idéologie et les déficits non avoués » ${ }^{19}$ de nos sociétés modernes. Le lecteur, lui, se délecte de cette confrontation avec les divinités du paysage classique européen grec et allemand : le débat animé entre le couple Zeus/Mars (pour la tradition grecque) et Marx/Engels (représentants quasi obligés de l'idéologie socialiste dans le contexte de la RDA), constitue l'un des grands moments du jeu et du roman. La « conscience chrétienne » européenne n'est pas non plus oubliée dans l'épilogue, puisque Kramer va même croiser l'archange Gabriel. Ultime pied-de-nez à l'histoire de notre civilisation, ce dernier ne jouit que du statut d'invité au milieu de tous ces puissants, mais il est chargé d'expliquer à Kramer qu'il va avoir le privilège de jouer une nouvelle partie en pleine conscience de son nouveau statut, configuration exceptionnelle jamais testée auparavant, occasion unique de mesurer chez l'homme le conflit entre pouvoir et conscience.

Le roman se termine sur une note désabusée, sur un Nachspiel, à la fois épilogue et ultime partie accordée au moraliste Kramer, condamné

18. - « Du bist Software. Du bist ein Programm, Rüdiger, erschaffen um mit uns ein Spiel zu spielen. Das Spiel heißt Polyplay. Es ist nicht das kleine, dumme Spiel, das du bisher kanntest [...]. Es ist ein viel größeres, viel komplexeres, viel wunderbareres Spiel, als du dir überhaupt vorstellen kannst. Es ist eine Welt. Viele Welten. Wir sind Spieler. Wir sind Götter. Und du bist Teil des Spiels. Ein intelligenter, fühlender Teil des Spiels. Eine Schachfigur mit Gehirn », PolyPlay, ibid., p. 169.

19. - «SF könnte eine gesellschaftliche Satire im Angriff sein, wenn mit Angriff nicht der platte Aufruf zu sozialem Aktivismus verbunden ist, sondern der Angriff auf Mythen, Ideologie, verleugnete Missstände ». Interview de Marcus Hammerschmitt avec Jakob Schmidt, op. cit., p. 1. 
à vivre par le logiciel et qui va tenter de défendre son libre-arbitre en dépit de son statut de "machine », autrement dit : comment repenser la question du déterminisme. La référence à la littérature et à l'esprit baroque va même plus loin dans PolyPlay puisque les « dieux » reconnaissent eux-mêmes que leur expérience scientifique n'est pas dénuée d'aspects ludiques et que la particularité remarquable du programme Polyplay est d'allier l'agréable à l'utile, traduction littérale de la maxime baroque, devise des auteurs du XVII siècle allemand : «prodesse et delectare » - une définition de la littérature dont se réclame Grimmelshausen dans le prologue du Simplicius sous la forme d'un texte annoncé comme " amusant et utile à lire/manniglich lustig und nützlich zu lesen », définition qui pourrait aussi figurer en exergue du roman de M. Hammerschmitt.

Roman policier à l'esprit résolument baroque comme le confirme le thème du jeu qui est au cœur du schéma narratif, PolyPlay tient plus de la satire que du simple divertissement uchronique. Témoin de l'une des ruptures majeures de l'histoire européenne au XXe siècle, l'effondrement du bloc communiste, M. Hammerschmitt envisage, traduit et finalement dénonce cette rupture par l'écriture. À la fois parodie littéraire et critique sociale, le cyberthriller de $\mathrm{M}$. Hammerschmitt répond à un devoir de mémoire imposé par la césure de l'automne 1989 comme le résume l'auteur :

Une des fonctions de PolyPlay relève de l'archéologie. Aussi curieux que cela paraisse, il faut aujourd'hui, douze ans après la « réunification », rappeler à certains qu'il a existé quelque chose du nom de RDA. Le livre comme critique de l'oubli ${ }^{20}$.

Mais la relecture uchronique du passé récent de l'Allemagne conduit le lecteur à une réflexion plus générale sur la place de l'homme dans l'univers, thématique ontique par excellence des ouvrages d'anticipation $^{21}$. Par le biais de l'uchronie, l'auteur nous emporte dans une spirale infernale qui bouscule et bouleverse de manière tout à fait inattendue notre rapport à l'histoire récente. Au-delà d'un ancrage historique propre à l'espace allemand, dont la gestion du passé est implicitement dénoncée par l'argument de départ, ce roman policier futuriste remet en cause de manière radicale notre rapport individuel au temps humain, ques-

20. - « Eine der Aufgaben von PolyPlay ist eine archäologische. So seltsam es klingt: Manche müssen heute, zwölf Jahre nach der 'Wiedervereinigung', daran erinnert werden, dass es so etwas wie die DDR überhaupt gab. Das Buch als eine Kritik des Vergessens ». Interview de Marcus Hammerschmitt avec Jakob Schmidt, ibid., p. 2.

21. - Alexandre Hougner, Science-fiction et société, Paris, P.U.F., 2000. 
tionnant sur un mode aussi ludique que métaphysique la fragile réalité temporelle de nos existences.

Si le genre du polar a réussi depuis deux décennies au moins à gagner un public de plus en plus nombreux outre-Rhin sous l'impulsion de la vogue nordique qui a envahi l'Allemagne comme le reste de l'Europe, c'est à travers un mélange particulier de suspense psychologique teinté d'enjeux politico-historiques. L'enquête policière du lieutenant Kramer dans PolyPlay se double de la propre enquête du lecteur désireux de percer le mystère du dérèglement de la chronologie - si bien qu'une double enquête se noue dans ce polar uchronique, témoin de l'omniprésence de l'histoire récente dans la mémoire collective de l'Allemagne unifiée, et qui se révèle une efficace satire sociale tout autant qu'une réponse aux bouleversements et ruptures de l'automne 1989. 
